\title{
Detection and Contribution of Outliers for Subjective Evaluation of Sound
}

\author{
Samir N. Y. Gerges \\ Federal University of Santa Catarina (UFSC), Mechanical Engineering, Florianopolis, SC, Brazil 88040-900. \\ Institute Federal de Santa Catarina (IFSC) / Mechatronics, Florianopolis, SC, Brazil 88020-300. \\ NR consultancy and Training, Florianopolis, SC, Brazil 88035-200.
}

Roberto A. Dias

Institute Federal de Santa Catarina (IFSC) / Mechatronics, Florianopolis, SC, Brazil 88020-300.

\author{
Rafael N. C. Gerges \\ NR consultancy and Training, Florianopolis, SC, Brazil 88035-200.
}

(Received 8 February 2015; accepted 3 May 2016)

The subjective evaluation of noise perception is a very broad topic that has many applications in the field of acoustics. Large variability is usually associated with a subjective evaluation that appears in the standard deviation. This is due to a small amount of subjects (the outliers), who had different responses compared to most of the other subjects. By using the Bootstrap statistical method, this paper shows how to identify the outliers and quantify the contribution to the final results with and without considering the outliers in the calculation.

\section{INTRODUCTION}

The subjective evaluation of noise perception plays an important role in the decision making of many applications in the field of acoustics, such as the evaluation of noise perception (annoyance) in communities located near airports and studies on traffic noise, product sound quality, environmental soundscapes, sleep disturbance, and hearing protector noise attenuation. The subjective perception of noise by a group of human evaluators usually shows a large variability, as observed through the standard deviation. This is because the subjects differ in terms of their experience, attitudes, expectations, age, personal state of mind, sensitivity to noise, fear of harm connected with the source, personal evaluation of the source, coping capacity with respect to noise, trust in or lack of confidence in the relevant authorities, and a history of noise exposure, among other factors. Some subjects paid greater attention to the assessment and provided more accurate responses while others did not concentrate properly on the task and performed the evaluation simply for the payment. In general, a small amount of the subjects gave responses that differed from most of the other subjects. These few subjects tended to have a considerable influence on the final results and were the main reason behind the high standard deviation. Thus, they will be considered as "outliers." This is a very broad topic that has extensive applications in different fields. ${ }^{1-3}$

\section{STATISTICAL DETERMINATION AND DE- TECTION OF OUTLIERS}

There were different methods available for the detection of outliers, ${ }^{4}$ including the box plots, miss match models, the Dixon test, the Grubbs test, and Z-scores. In the study reported, a mismatch model and the bootstrap method were used to investigate the statistical distribution and to identify outliers.
Additionally, the quantitative effect on the final results when the outliers were removed from the dataset was determined.

The subjects considered as outliers, who generally represented around 3 to 5 out of the total 20 to 30 subjects in the cases presented herein, were not true outliers. However, their subjective evaluation was very different from that of the other subjects. The objective of this paper is to describe a way to detect these outlier subjects and evaluate their effect on the final results by eliminating them. Some real cases were described here to show the application of the bootstrap statistical technique to the identification of outliers and to evaluate their contribution to the results.

\subsection{Bootstrap Method}

The bootstrap method was introduced by B. Efron in $1979^{5}$ and its use in statistical sciences became widespread within a few decades. This method involves taking the original dataset of $N$ elements and sampling from it by using a computer in order to generate a new sample with size $N / 2$. The elements are then exchanged randomly between these two datasets (each of size $N / 2$ ).

This process is repeated many times and for each of these bootstrap samples, the final parameter (e.g. the mean) is computed. The histogram of this final parameter is obtained with the mean value and standard deviation together with the contribution of the $N$ original elements to the final parameter. The statistical distribution is then observed and some outliers will show a non-Gaussian distribution. The outliers can also be identified and their contribution to the final results evaluated. If these outliers are eliminated and the distribution is recalculated, it becomes more Gaussian with a better estimation of the parameters.

In this study, 20 subjective responses were obtained, with 3 to 7 questions given to each subject. The random selection 


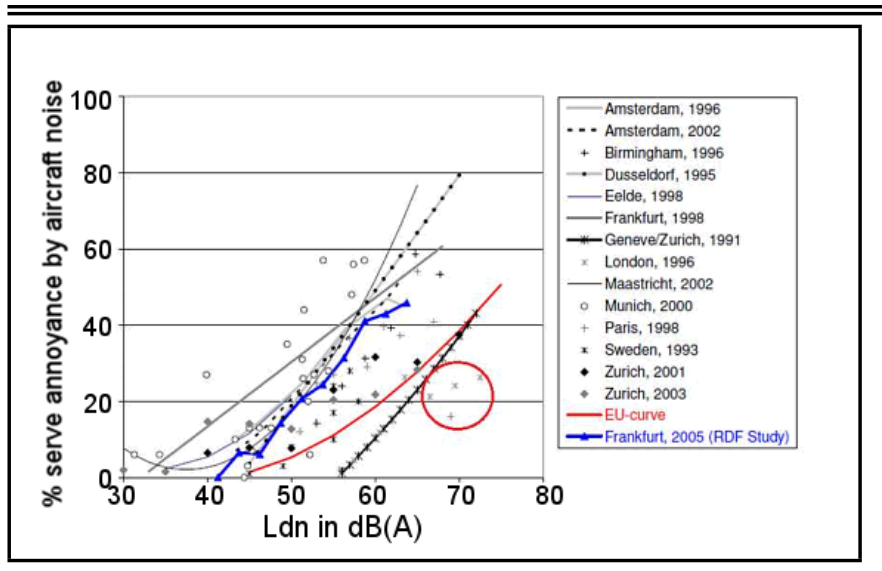

Figure 1. Dose-response data for severe aircraft noise annoyance from several surveys using a cut-off point of 70 to $75 \%$ of the response scale for the definition of high annoyance (HA). Note that the four points (in the red circle) with an annoyance of less than $30 \%$ were considered as "outliers".

of 10 subjects (out of 20) was applied. The procedure was repeated 20,000 times for each subject. The results were then used to identify the outliers and their contribution to the final result.

\section{SUBJECTIVE ASSESSMENT: RESULTS WITH OUTLIERS REPORTED IN THE LITERATURE}

A large number of publications reporting subjective surveys demonstrate that some subjects (outliers) are only very slightly disturbed by high levels of noise exposure. Some of these cases are described herein. Figure 1 shows the percentage of severe annoyance caused by aircraft noise as a function of the noise level (Ldn in dBA) ${ }^{6}$ for different airports. It is clear that there are a few subjects with annoyance below $30 \%$ (marked with a circle below the line). These were considered as outliers since they represented a small percentage of the total number of subjects and their values differed greatly from those of the other subjects. This example proved that a few subjects could typically alter the final results if they were considered in the statistical evaluation. These few outliers usually gave the response that the noise from the airport was not very annoying. This could be because they had a vested interest in the airport. For instance, they may own a business inside the airport and thus wished to see its level of activity and capacity maintained or even increased.

Another published example can be seen in Fig. 2, which shows the percentage of highly sleep-disturbed subjects as a function of the sound pressure levels (LeqA) of road traffic noise. $^{7}$ In this case, there was one (marked X) with a high level of exposure (70 dBA) and low disturbance (10\%). This point on the curve was certainly an outlier where perhaps the subject did not pay due attention and provided an inaccurate answer or perhaps this subject had a vested interest in traffic noise. For example, they may have a commercial concern.

The following are four cases of different applications, which demonstrated how to identify outliers, delete them, and quantify the effect of their contribution to the results.

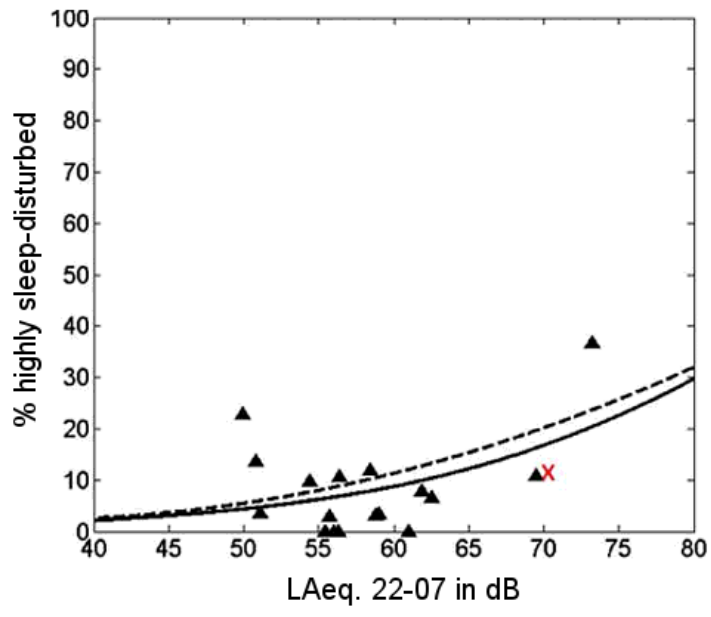

Figure 2. The percentage of the highly sleep-disturbed population (\%HSD) as a function of LAeq.22-07 in response to road traffic noise. The solid line and the data points in the LAeq.22-07 range and 49 to $73 \mathrm{dBA}$ represent the CENVR results. The dashed line represents the European research result. ${ }^{7}$ The point $\mathrm{X}$ indicates an outlier.

\subsection{Case Study 1: Hearing Protector Noise Attenuation Measurements}

Measuring the noise attenuation of hearing protector devices (HPDs) using the REAT "Real-ear Attenuation at Threshold" method was based on subjective measurements, where each subject determines their open (without HPD) and closed (with HPD) threshold levels. ${ }^{8,9}$ The subjective determination of the threshold levels showed a high variation between the subjects, even when they were qualified and familiarized with the method used to determine these threshold levels, as required by the relevant standard. Some subjects paid greater attention and could determine their threshold with more accuracy than others. Some subjects simply did not pay attention and answered randomly depending on their mood and mental condition that day. This paper shows a methodology to observe the statistical distribution and quantify the contribution of each subject to the final single number Noise Reduction Ratio (NRRsf). ${ }^{8,9}$ Eliminating a few subjects (the outliers) increased the NRRsf and reduced the variability of the measurements (from around \pm 4 to \pm 1 ). The results for the measurement of 20 different brands of pre-molded earplugs were reported.

Hearing protector noise attenuation measurements were taken according to ANSI 12.6-2008 ${ }^{8}$ and ISO 4869-5/4869$1,{ }^{9}$ where the hearing threshold of a number of subjects were measured with and without the use of a hearing protector and the difference gave the noise attenuation. Two fitting methods were considered in the standards: (i) supervised fitting (method A as described in ANSI S12.6-2008 or ISO 48691 ), and (ii) subject fitting (method B as described in ANSI S12.6-2008 or ISO 4869-5). There was a high variability in the results obtained for the same HPD in the same laboratory due to the hearing protector fitting, especially for earplugs (compared with earmuffs). This measurement variability was higher for inexperienced subjects (method B) than for trained subjects (method A) and could reach $\pm 3 \mathrm{~dB}^{8}$ or even a higher value. According to ANSI S12.6-2008, it was necessary to acquire data for at least twenty subjects for earplugs and ten subjects for earmuffs. Some subjects did not pay due attention to 
specifying their thresholds. In some cases, a few subjects with threshold levels that differed from the majority of the subjects could considerably alter the final noise attenuation values (by more than $\pm 3 \mathrm{~dB}$ ). That is, the Noise Reduction Ratio (NRR) and NRRsf (calculated using one standard deviation). Considering the calculation method and the standard deviation for the NRRsf values, the statistical interpretation of this value was under the conditions observed with the use of at least $84 \%$ of the user population; the attenuation was at least equivalent to the NRRsf value. The ISO 4869-2 standard stated that a variation of less than $\pm 3 \mathrm{~dB}$ was insignificant, but this was not supported by detailed research studies and was considered in most publications without the effect of the outlier subjects being investigated. This paper described how to identify these outlier subjects, that is, those with very different results compared with most of the subjects, and investigated the effect of eliminating them on the final NRRsf value. In a real situation in the field, most HPD users received training on each type of device and they were aware of the risk of permanent hearing loss if the HPD was not properly fitted and used throughout all work shifts. Therefore, the presence of these outliers could inhibit an evaluation of the real situation and it could be useful to consider their elimination from the final results in order to obtain a truly representative sample.

The Real-Ear Attenuation at Threshold (REAT) method was the gold-standard method, which was most commonly used and accepted worldwide for the measurement of hearing protector noise attenuation. This was a subjective measurement where the subjects determine their own threshold levels (with and without an HPD). The accuracy of this measurement was strongly dependent on the subject's perception of the sound level at the ear and each subject had to concentrate to determine their own threshold level. Considering that the subjects were paid, earning between 10 to 50 USD for each test, there was no guarantee that they had properly determined their threshold level. Some subjects paid greater attention than others and some may have had work experience and/or some education that allowed them to provide better results. Therefore, for each hearing protector brand measurement, especially for plug-type devices (which were more difficult to fit than earmuffs), there were sometimes a few subjects (generally not more than five) who showed a low accuracy in determining their threshold levels, which could result in large variations in the NRRsf value. In this paper, the results that were obtained for 20 different premolded earplug brands by using the subject fitting method (B) of ANSI S12.6-2008 based on the evaluations of 20 subjects were reported and analyzed.

In this section, the statistical distribution for each HPD brand is determined and a method is shown to detect the outliers and observe the final results for the NRRsf after eliminating the small number of outliers.

\subsection{Methodology}

Twenty brands of earplugs were tested by using the bootstrap statistical methodology. For each group of the ten subjects (out of a total of 20 subjects), the NRRsf was calculated and this procedure was repeated 100,000 times for each HPD in order to plot the statistical distribution with high resolution.

As an example, the following table shows the results for 20 subjects for one brand of pre-molded earplugs and two mea-

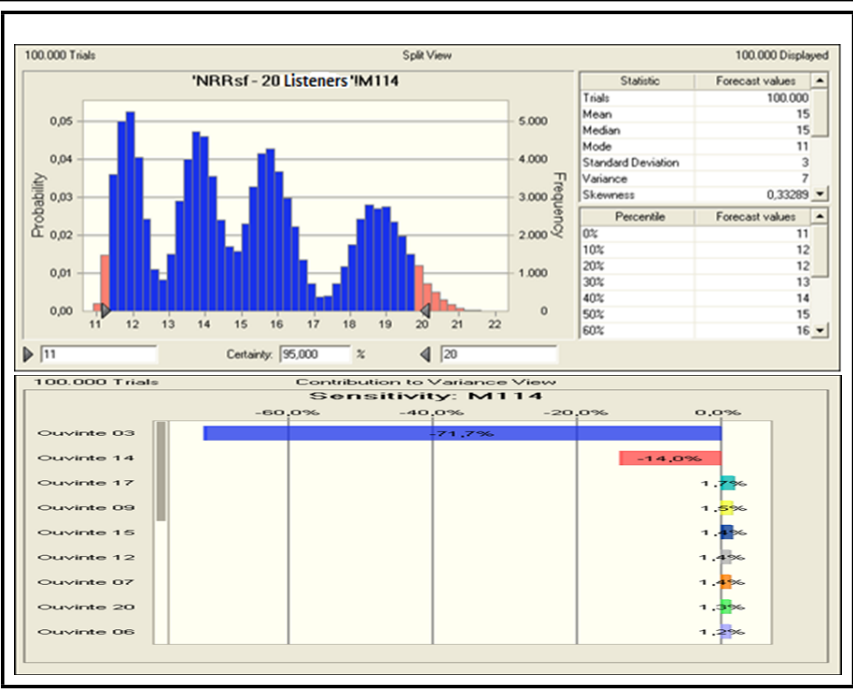

Figure 3. The statistical distribution of NRRsf and the contribution of subjects 3 and 14.

surements (open and closed thresholds) being taken for each subject. The noise attenuation results are shown in Table 1 for the 20 subjects with two repetitions for each subject.

Tabel 1 in A1 shows the results for 20 subjects, with two measurements each (open and closed thresholds) for an earplug HPD. Figure 3 shows the results for the bootstrap statistical analysis, considering 10 subjects, with 100,000 repetitions. The statistical distribution for the NRRsf shows a complex distribution, with four peaks for a range of NRRsf values of 11 to 20 . In this case NRRsf=15 with a standard deviation of $3 \mathrm{~dB}$ (right side of Table 1).

The Crystal Ball software was used to evaluate the sensitivity of the result with respect to each subject. Fig. 3 shows that Subject 3 contributes $71.7 \%$ to the NRRsf value and Subject 14 contributes $14 \%$.

On removing Subjects 3 and 14 and recalculating the statistical distribution, a new distribution, which was very close to Gaussian, was obtained, as shown in Fig. 4. Additionally, he NRRsf value increased from 15 to $19 \mathrm{~dB}$, while the standard deviation decreased from \pm 3 to $\pm 1 \mathrm{~dB}$. This process could then be repeated and in Fig. 4, it can be observed that there was still a $27 \%$ contribution from Subject 16, $15 \%$ from Subject 10, and $13 \%$ from Subject 1. It was recommended that in order to keep the results as representative as possible of a real situation, a limited number of subjects were removed, where very few users were not able to achieve a proper fitting of the HPD. Therefore, in this study, the removal was limited to not more than $30 \%$ of the effect on the NRRsf value.

In this study, Case 1 shows clearly that by observing the statistical distribution, calculated for each group of 10 subjects and repeated 100,000 times, it is possible to detect the extent to which the results deviate from a Gaussian distribution. Crystal Ball software was then used to identify the contribution of each subject to the NRRsf. With the removal of only two subjects in Case 1, the NRRsf increased from 15 to $19 \mathrm{~dB}$ and the standard deviation decreased from 3 to $1 \mathrm{~dB}$.

\subsection{Case Study 2: Noise in the Vicinity of an Airport}

A study was carried out in a residential area near an airport. Twenty subjects were interviewed and each was given 7 ques- 


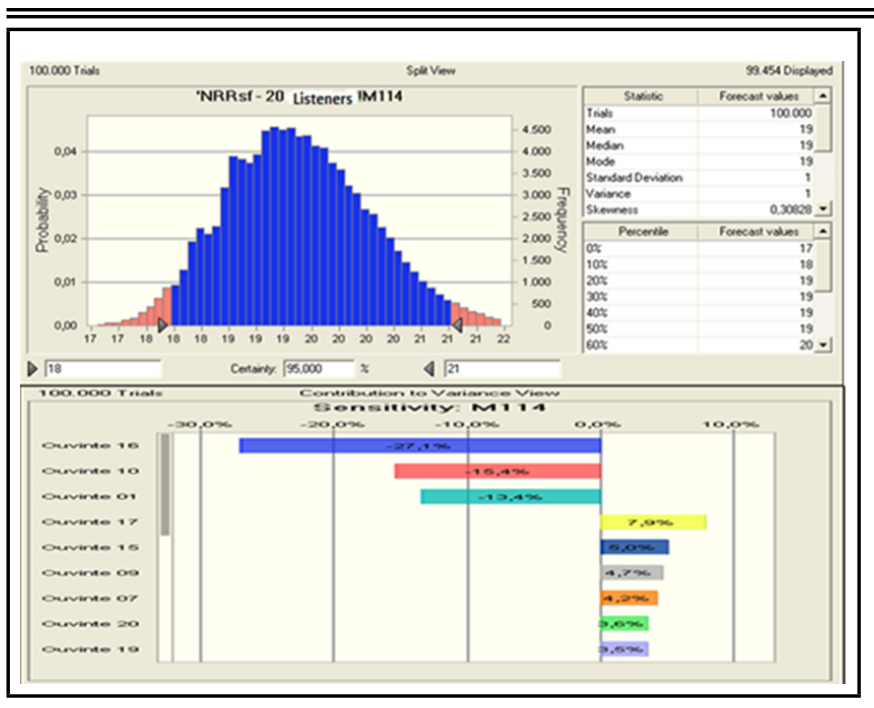

Figure 4. Case 1 after removing the outliers (Subjects 3 and 14), which show the NRRsf increased from 15 to $19 \mathrm{~dB}$ and the standard deviation decreased from 3 to $1 \mathrm{~dB}$.

tions as follows:

1. Considering approximately the past hour, how much did aircraft noise as a whole bother, disturb, or annoy you?

Please give a rank between extremely annoying (0) and not at all (10).

2. How much does aircraft noise disturb you while you are watching TV?

Please give a rank between extremely disturbing (0) and not at all (10).

3. How much does aircraft noise disturb you during conversations with others?

Please give a rank between extremely disturbing ( 0$)$ and not at all (10).

4. How loud was the last aircraft sound?

Please give a rank between extremely loud (0) and not loud at all (10).

5. How much does aircraft noise disturb you while you are reading?

Please give a rank between extremely disturbing (0) and not at all (10).

6. How much does aircraft noise disturb you while you are sleeping?

Please give a rank between extremely disturbing (0) and not at all (10).

7. Would you like to move away from the airport to avoid aircraft noise and if so, how far would you like to be?

Please give a rank between I would like to be very near to the airport (e.g. because it is cheaper) (0) or far away (10).

These seven questions were given to 20 subjects, who were chosen randomly at a residential area around an airport. The obtained scores from their responses are shown in Table 2, A2.

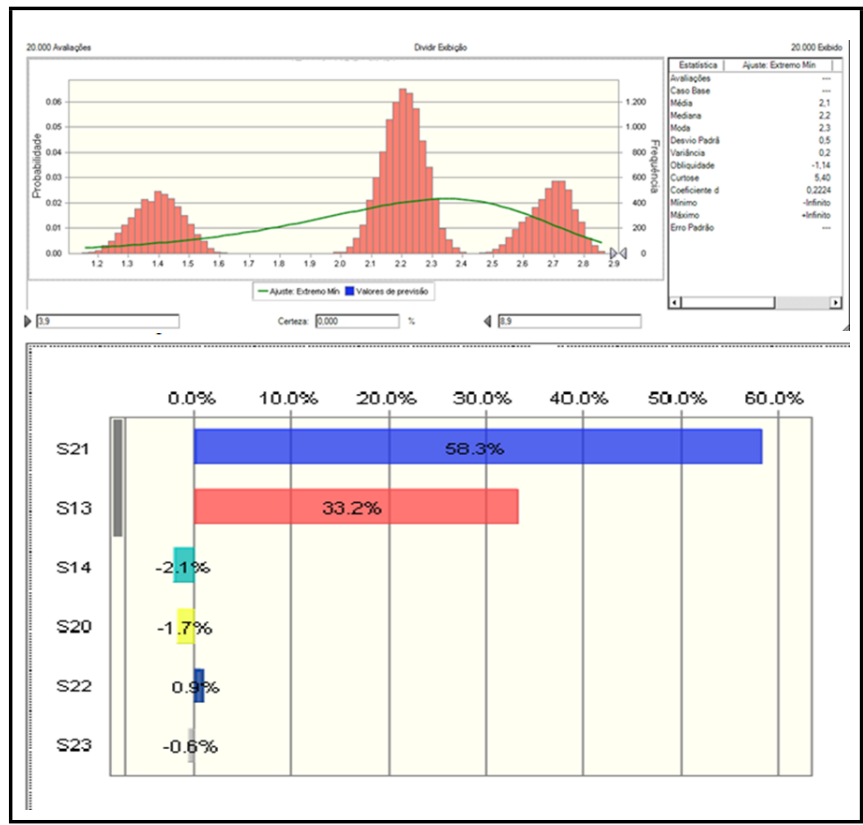

Figure 5. The bootstrap statistical results.

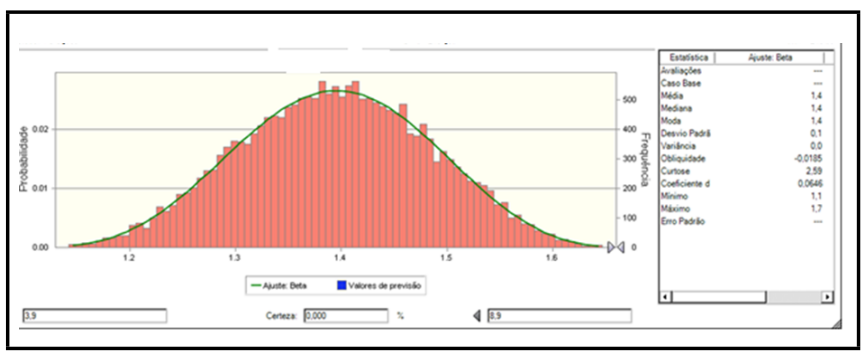

Figure 6. Case 2 after removing the outliers.

Note that a low score for the responses means that the noise was incredibly annoying to the subject.

Analysis carried out applying the bootstrap statistical technique using Crystal Ball software for the data shown in Table 2, A2, provided the results given in Fig. 5. The mean value obtained was 2.1 and the standard deviation was 0.5 . Three peaks were observed in the statistical distribution. Also, two subjects (S21 and S13) were identified as having the largest contributions of $58.3 \%$ and $33.2 \%$, respectively.

The two aforementioned subjects were removed and the analysis applying the bootstrap statistical technique was repeated. For the new results without these two outliers the mean value was 1.4 and the standard deviation was 0.1 , while the statistical distribution was very close to normal, as shown in Fig. 6. Therefore, in this case, on identifying the outliers and removing them, the mean decreased from 2.1 to 1.4 and the standard deviation from 0.5 to 0.1 .

Case 2 shows clearly that by observing the statistical distribution, calculated for each group of 10 subjects out of a total of 20 subjects, with 100,000 repetitions, it was possible to detect the normality of the results and the deviation from a Gaussian distribution. The Crystal Ball software could then be used to identify the contribution of each subject and detect two subjects with a large contribution to the final results. By removing these two outliers, the final results better represent the real situation (a high level of annoyance). 


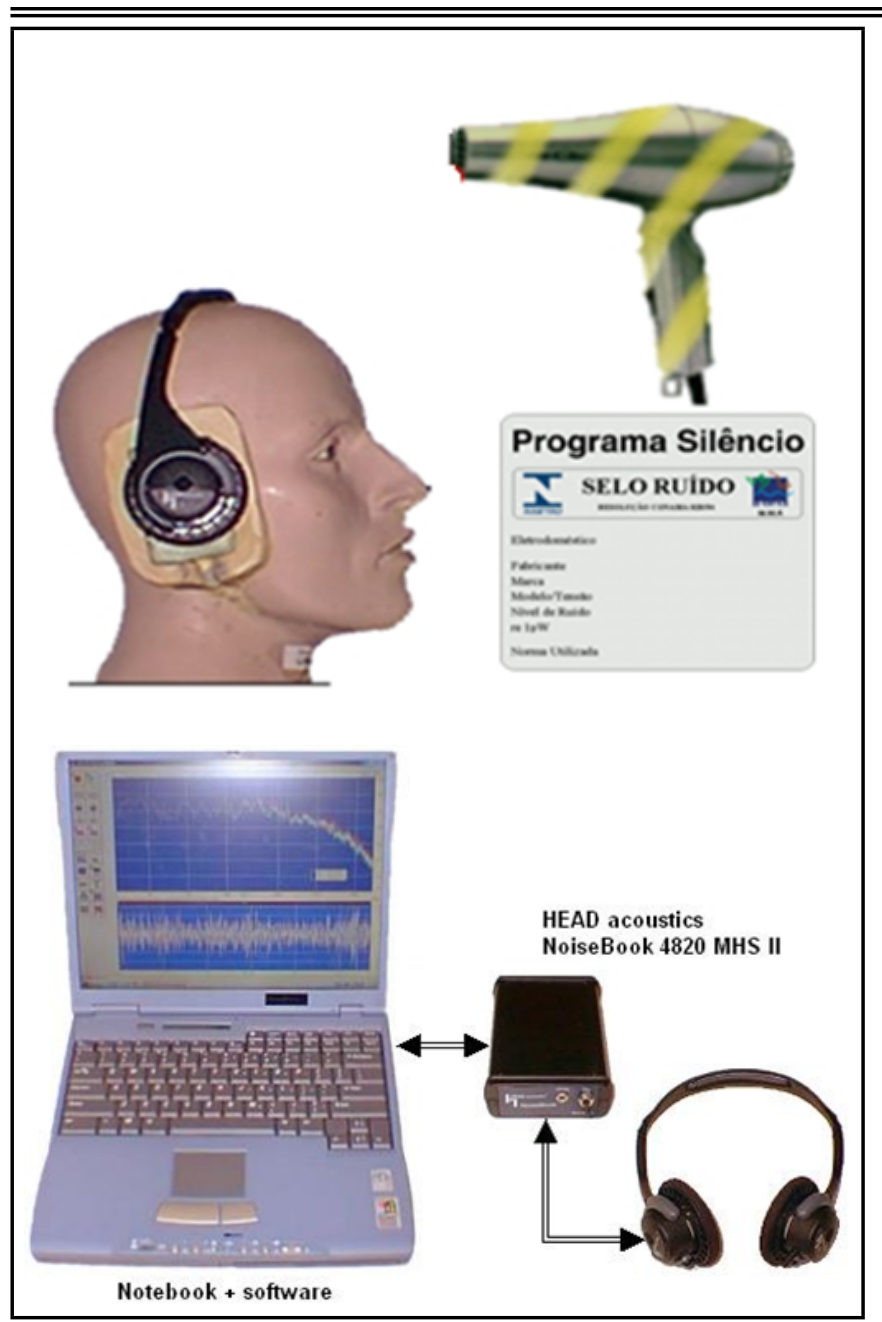

Figure 7. The binary recording of the sound using NoiseBook.

\subsection{Case Study 3: Product Sound Quality Evaluation}

Three different brands of hair dryers of 1100 watts each were evaluated in terms of noise quality. Special sound quality equipment for recording and playback, called "NoiseBook" produced by HEAD Acoustics, was used to binaurally record the noise and play it back to an evaluation jury (see Fig. 7).

The sound quality metrics parameters calculated were the sound pressure levels in $\mathrm{dB}$ and $\mathrm{dBA}$, the loudness and the sharpness (see Fig. 8).

The results for the measurements shown in Fig. 8 indicate that hairdryer $\mathrm{A}$ is probably the least noisy, followed by $\mathrm{C}$, and then $\mathrm{B}$. The difference between $\mathrm{B}$ and $\mathrm{C}$ is very small and these two hairdryers can thus be graded as similar.

A subjective evaluation was carried out by using a panel of 20 women. Each answered the following three questions:

1. After listening through the binaural headset to the sound of each hairdryer (A, B and then C), please select a score between 0 (low noise) to 10 (very noisy).

2. After listening through the binaural headset to the sound of each hairdryer (A, B and then C), please select a score between 0 ("I would like to purchase it") to 10 ("I would not like to purchase it").

3. After listening through by the binaural headset to the sound of each hairdryer (A, B and then C), please select

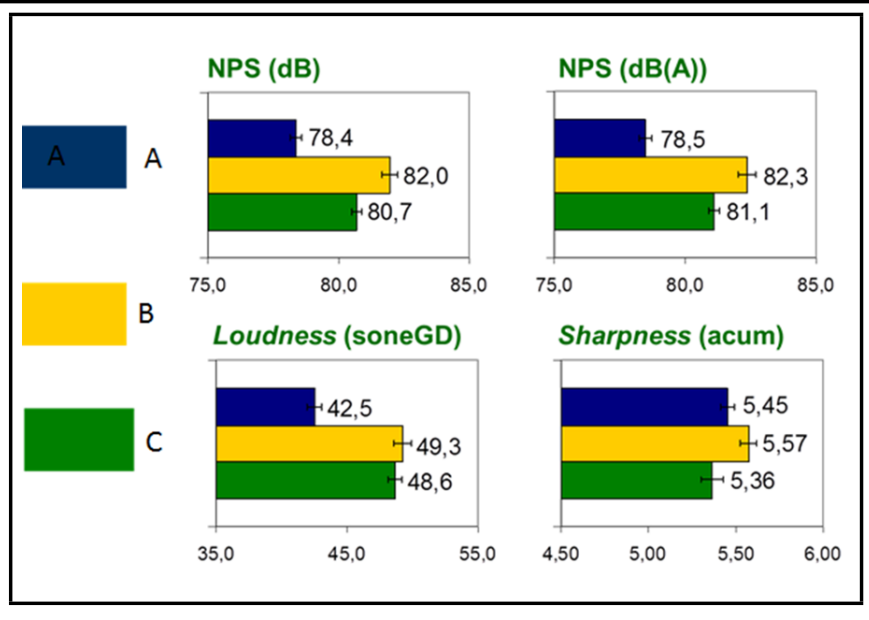

Figure 8. The sound quality parameters, dB, dBA, loudness, and sharpness for the three tested hairdryers.

a score between 0 (very efficient dryer) to 10 (very inefficient dryer).

Table 3, A3, shows the scores obtained in the subjective evaluations of 3 questions for dryer $\mathrm{A}, \mathrm{B}$, and $\mathrm{C}$.

Figure 9 shows the results obtained from the bootstrap calculation for the statistical distribution, mean and standard deviation and the contribution of outlier subjects in the case of hairdryers A.

From the results shown in Fig. 9, it can be observed that after removing the outliers, the mean score for the best hairdryer (A) was 1.1 with a standard deviation of 0 (rounded). Hairdryers $\mathrm{B}$ and $\mathrm{C}$ appeared to be similar with mean values of 1.2 and 1.5 , respectively. Once again, in this example, the potential of the bootstrap technique for the identification of outliers and demonstrate their contribution to the final results was verified.

\subsection{Case Study 4: Traffic Noise and Sleep Disturbance}

Noise was perceived by a specific auditory system in humans. Therefore, it was a phenomenon that was sensed and evaluated by everybody and this was why exposure to noise was one of the most common complaints, if not the most frequent complaint, of people living in large cities. In these areas and their surroundings, the most frequently cited sources of noise were traffic, followed by neighborhood noise, and then aircraft noise. Sleep is a physiological state that needs to be properly achieved to allow a living organism to recuperate normally. This state is sensitive to environmental factors that can interrupt it or reduce its duration. Ambient noise, for example, is comprised of external stimuli that are processed by a sleeping persons sensory functions, with a non-conscious perception of their presence. Over the past 30 years, research into environmental noise and sleep has focused on different situations and environments and therefore the findings are variable. In this regard, some fundamental questions remain to be answered regarding the perception of noise by communities living near roads which receive heavy traffic. A large number of subjective studies have been published in the literature, but again subjective evaluations lead to large standard deviations due to extreme responses by some subjects.

A high class residential area in the city of Florianopolis, Santa Catarina State, in the south of Brazil was evaluated for 


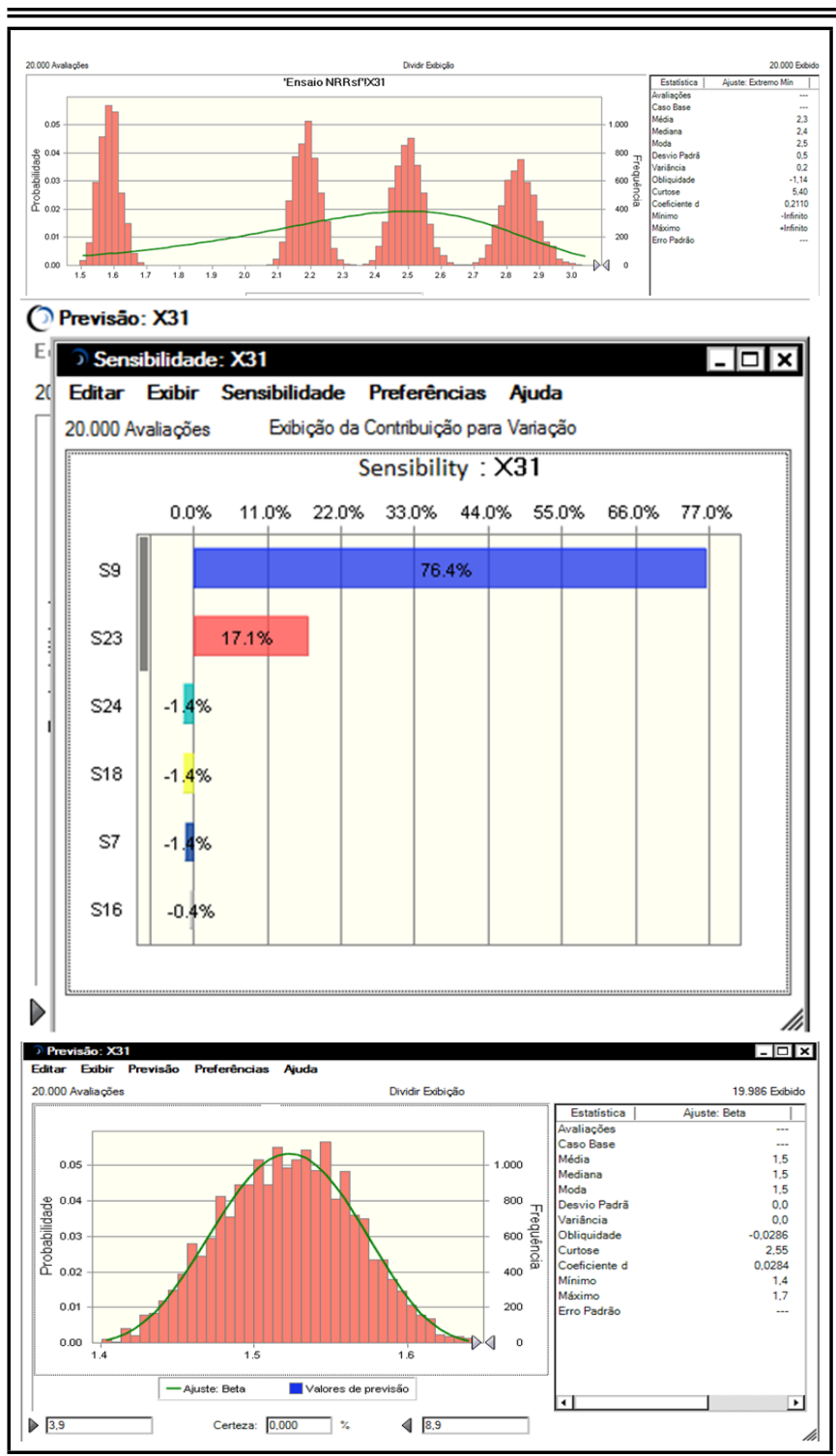

Figure 9. The bootstrap statistical results for hairdryer A with outliers (the top curve) and without outliers (the bottom curve).

sleep disturbances. This residential area has eight traffic lanes and the residents mostly overlook a water front and a leisure walking track and cycle lane. The high density of the traffic results in sleep disturbance within the community. Research was carried out through interviews with community members who answered a questionnaire with the following five questions:

1. Do you suffer sleep disturbance due to traffic noise?

Please give a rank between extremely difficult to sleep (0) and easy to sleep (10).

2. Do you have trouble conversing at home because of traffic noise?

Please give a rank between extremely difficult (0) and easy to converse (10).

3. Do you have problems listening to TV programs at home because of traffic noise?

Please give a rank between extremely difficult (0) and easy to listen (10).

4. Do you experience problems doing homework or studying because of traffic noise?

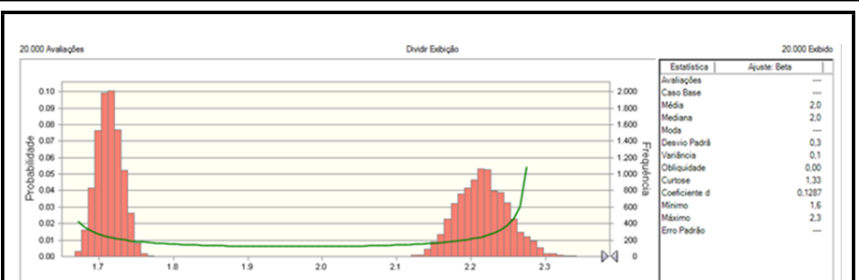

Sensibilidade: X31

$-|\square| x \mid$

Editar Exibir Sensibilidade Preferências Ajuda

20.000 Avaliações Exibição da Contribuição para Variação

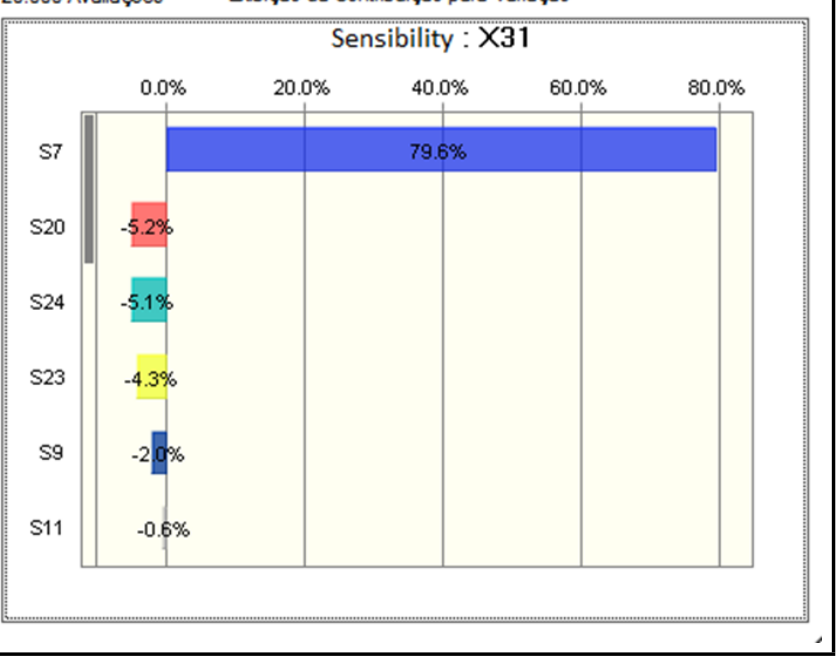

Figure 10. The bootstrap statistical results for traffic noise with outliers and its contribution.

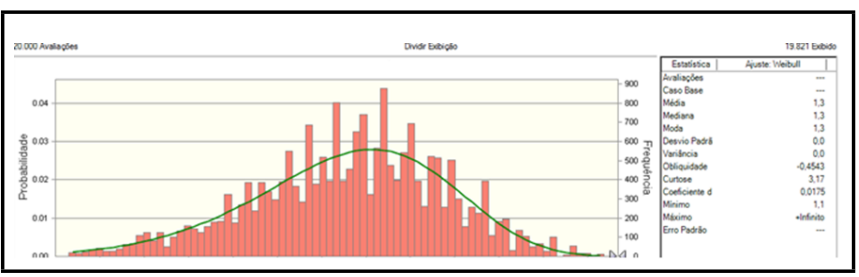

Figure 11. The bootstrap statistical results for traffic noise without the outliers

Please give a rank between extremely difficult (0) and easy to study (10).

5. Do you have a problem relaxing and thinking because of traffic noise?

Please give a rank between extremely difficult (0) and easy to relax (10).

Table 4, in A4, shows the scores between zero and ten given by twenty subjects. A low score means high traffic noise.

The data in Table 4 were fed into the bootstrap framework, the mean values and standard deviations were calculated, and the statistical distribution was observed. The results identified the outliers and indicated their contribution. After removing the outliers, the statistical distribution came very close to Gaussian and the results were refined. Figure 10 shows the statistical distribution and the contribution of the outliers and Fig. 11 shows the statistical distribution after removing the outliers.

Once again, in this case applied to a high level of traffic noise, it was possible to remove an outlier through the use of the bootstrap statistical method and obtain better results. 


\section{CONCLUSIONS}

This paper demonstrates that with the use of the bootstrap statistical method, it is possible to detect abnormalities in subjective data and after removing outliers, the results show a normal statistical distribution with more accurate final estimates. Four case studies have been presented: noise attenuation measurements for a pre-molded earplug hearing protector, the annoyance of noise from an airport as perceived by the community in a neighboring residential area, an evaluation of the sound quality of hairdryers, and sleep disturbance due to traffic noise. This methodology can be used in many other cases involving the analysis of subjective sound perception.

\section{ACKNOWLEDGEMENTS}

This research was carried out with the support of the Brazilian funding bodies for research (CNPq, CAPES, and FINEP) and the Laboratory of Personal Protection Equipment (LAEPI) of NR Consultancy Ltd. The technical support by Rodrigo Mazza from Honeywell, with regard to the bootstrap statistical technique, was greatly appreciated.

\section{REFERENCES}

1 Karanjit Singh and Suchita Upadhyaya. Outlier detection: applications and techniques. International Journal of Computer Science Issues, 9 (1), 307-323, (2012).

2 Jinhong Yang, Tingquan Deng, and Ran Syui. An adaptive weighted one-class svm for robust outlier detection. Proc. of The 2015 Chinese International System Conference, 475-484, (2015).

3 Joel W. Branch, Chris Giannella, and Bloeslaw Szymanski. Ran Wolff and Hillol Kargupta. In-network outlier detection in wireless sensor networks. Knowledge and Information Systems, 34 (1), 23-54, (2012).

4 Barnett, Vic and Lewis, Toby. Outliers in Statistical Data, John Wiley \& Sons, (1994).

5 Efron, B. Bootstrap methods: another look at the jackknife, Ann. Statist. 7, (1), 1-26, (1979).

6 Van Kempen EEMM, Van Kamp I. Annoyance from Air Traffic Noise. Possible Trends in Exposure-Response Relationships. RIVM; Bilthoven, The Netherlands: 2005. Report 01/2005 MGO EvK, Reference 00265/2005.

7 Miedema H. M. E. Oudshoorn CG. Annoyance from transportation noise: Relationships with exposure metrics DNL and DENL and their confidence intervals. Environ. Health Perspect. 2001(109), 409-416, (2001).

8 ANSI S12.6-2008 - Methods for Measuring the Real-Ear Attenuation of Hearing Protectors.

\section{APPENDIX 1}

Table 1. Noise attenuation results for 20 subjects, with two measurements each (open and closed thresholds) for an earplug HPD.

\begin{tabular}{|c|c|c|c|c|c|c|c|}
\hline \multicolumn{8}{|c|}{ Hearing Protector Noise attenuation $(\mathrm{dB})$} \\
\hline Subject & 125 & 250 & 500 & 1000 & 2000 & 4000 & 8000 \\
\hline \multirow{2}{*}{1} & 19.17 & 17.00 & 21.00 & 22.67 & 27.83 & 19.00 & 28.83 \\
\hline & 14.50 & 18.67 & 21.33 & 22.50 & 27.83 & 19.33 & 39.17 \\
\hline \multirow{2}{*}{2} & 19.67 & 20.00 & 21.00 & 19.33 & 29.50 & 26.50 & 47.67 \\
\hline & 17.00 & 19.83 & 23.67 & 20.17 & 30.50 & 21.67 & 35.83 \\
\hline \multirow{2}{*}{3} & -0.83 & 3.67 & 2.50 & 6.50 & 5.50 & 12.50 & 6.50 \\
\hline & 5.83 & 2.00 & 6.67 & 5.17 & 6.17 & 12.00 & 1.67 \\
\hline \multirow{2}{*}{4} & 11.83 & 24.00 & 24.83 & 19.00 & 26.33 & 28.33 & 40.83 \\
\hline & 20.67 & 23.33 & 28.17 & 23.50 & 26.33 & 33.33 & 44.33 \\
\hline \multirow{2}{*}{5} & 16.67 & 17.33 & 16.67 & 16.83 & 28.67 & 34.50 & 40.17 \\
\hline & 16.00 & 18.00 & 22.67 & 24.50 & 23.67 & 26.83 & 37.17 \\
\hline \multirow{2}{*}{6} & 27.17 & 28.33 & 32.83 & 29.50 & 30.50 & 21.67 & 41.17 \\
\hline & 30.17 & 30.67 & 35.00 & 31.50 & 29.50 & 38.83 & 38.50 \\
\hline \multirow{2}{*}{7} & 21.83 & 22.00 & 16.67 & 22.00 & 27.33 & 23.33 & 26.67 \\
\hline & 29.17 & 31.67 & 35.67 & 26.00 & 28.83 & 34.50 & 46.17 \\
\hline \multirow{2}{*}{8} & 23.17 & 24.67 & 25.83 & 24.00 & 31.00 & 21.17 & 38.67 \\
\hline & 24.50 & 28.17 & 31.17 & 26.17 & 34.33 & 19.83 & 41.5 \\
\hline \multirow{2}{*}{9} & 22.00 & 25.17 & 30.33 & 29.67 & 26.67 & 31.00 & 42.00 \\
\hline & 26.67 & 21.33 & 29.33 & 28.67 & 28.67 & 29.67 & 41.33 \\
\hline \multirow{2}{*}{10} & 18.00 & 22.83 & 24.83 & 22.17 & 24.00 & 23.17 & 40.33 \\
\hline & 16.83 & 18.50 & 19.17 & 21.33 & 24.33 & 16.33 & 27.00 \\
\hline \multirow{2}{*}{11} & 13.83 & 14.67 & 10.83 & 15.17 & 25.00 & 23.17 & 32.67 \\
\hline & 22.50 & 25.67 & 28.00 & 24.67 & 38.50 & 30.00 & 50.83 \\
\hline \multirow{2}{*}{12} & 18.83 & 26.83 & 28.67 & 27.00 & 25.50 & 26.50 & 50.33 \\
\hline & 20.17 & 25.33 & 29.00 & 27.33 & 26.00 & 28.67 & 46.67 \\
\hline \multirow{2}{*}{13} & 17.67 & 18.55 & 23.33 & 22.55 & 30.17 & 25.50 & 31.00 \\
\hline & 5.50 & 4.83 & 10.17 & 13.55 & 28.33 & 20.50 & 29.00 \\
\hline \multirow{2}{*}{14} & 1.83 & 8.67 & 15.83 & 15.83 & 19.17 & 14.83 & 31.33 \\
\hline & 11.67 & 19.50 & 17.83 & 2.67 & 11.17 & 7.83 & 31.33 \\
\hline \multirow{2}{*}{15} & 25.83 & 24.00 & 25.17 & 23.83 & 32.00 & 38.83 & 38.17 \\
\hline & 29.67 & 27.83 & 27.83 & 24.00 & 31.83 & 31.17 & 40.17 \\
\hline \multirow{2}{*}{16} & 21.17 & 15.33 & 21.00 & 20.50 & 27.00 & 16.33 & 24.83 \\
\hline & 17.67 & 18.00 & 21.00 & 21.67 & 23.33 & 22.67 & 21.50 \\
\hline \multirow{2}{*}{17} & 30.50 & 25.83 & 30.67 & 25.00 & 28.83 & 32.83 & 47.17 \\
\hline & 28.50 & 27.33 & 30.17 & 27.00 & 32.33 & 29.17 & 45.83 \\
\hline \multirow{2}{*}{18} & 24.00 & 29.83 & 34.50 & 24.67 & 24.00 & 34.17 & 46.33 \\
\hline & 14.50 & 17.67 & 17.83 & 21.00 & 24.17 & 30.83 & 46.17 \\
\hline \multirow{2}{*}{19} & 20.17 & 20.33 & 26.33 & 21.17 & 23.17 & 26.50 & 45.00 \\
\hline & 24.00 & 24.67 & 29.00 & 21.83 & 28.00 & 44.33 & 51.17 \\
\hline \multirow{2}{*}{20} & 20.67 & 22.83 & 32.00 & 26.50 & 29.50 & 33.00 & 49.33 \\
\hline & 15.67 & 21.00 & 22.17 & 21.17 & 22.50 & 28.33 & 36.17 \\
\hline
\end{tabular}

9 ISO 4869, 1-5, Acoustics - Hearing protectors. 


\section{APPENDIX 2}

Table 2. Scores for the responses of 20 subjects to the 7 questions.

\begin{tabular}{||c|c|c|c|c|c|c|c||}
\hline Questions & 1 & 2 & 3 & 4 & 5 & 6 & 7 \\
\hline Subject 1 & 1.0 & 3.0 & 5.0 & 2.0 & 4.0 & 3.0 & 7.0 \\
\hline Subject 2 & 3.0 & 5.0 & 2.0 & 1.0 & 3.0 & 2.0 & 2.0 \\
\hline Subject 3 & 1.0 & 2.0 & 4.0 & 5.0 & 3.0 & 6.0 & 2.0 \\
\hline Subject 4 & 3.0 & 2.0 & 1.0 & 5.0 & 2.0 & 5.0 & 1.0 \\
\hline Subject 5 & 3.0 & 4.0 & 5.0 & 5.0 & 2.0 & 3.0 & 1.0 \\
\hline Subject 6 & 1.0 & 4.0 & 5.0 & 3.0 & 2.0 & 3.0 & 1.0 \\
\hline Subject 7 & 2.0 & 3.0 & 5.0 & 4.0 & 5.0 & 4.0 & 5.0 \\
\hline Subject 8 & 9.0 & 8.0 & 9.0 & 7.0 & 9.0 & 9.0 & 10.0 \\
\hline Subject 9 & 3.0 & 3.0 & 4.0 & 5.0 & 4.0 & 6.0 & 3.0 \\
\hline Subject 10 & 2.0 & 2.0 & 4.0 & 3.0 & 6.0 & 4.0 & 5.0 \\
\hline Subject 11 & 3.0 & 4.0 & 5.0 & 1.0 & 2.0 & 3.0 & 3.0 \\
\hline Subject 12 & 1.0 & 1.0 & 3.0 & 2.0 & 4.0 & 3.0 & 5.0 \\
\hline Subject 13 & 2.0 & 5.0 & 3.0 & 1.0 & 4.0 & 6.0 & 2.0 \\
\hline Subject 14 & 1.0 & 4.0 & 5.0 & 6.0 & 2.0 & 3.0 & 3.0 \\
\hline Subject 15 & 2.0 & 3.0 & 5.0 & 3.0 & 4.0 & 5.0 & 2.0 \\
\hline Subject 16 & 10.0 & 9.0 & 9.0 & 8.0 & 10.0 & 10.0 & 1.0 \\
\hline Subject 17 & 6.0 & 5.0 & 4.0 & 3.0 & 2.0 & 1.0 & 3.0 \\
\hline Subject 18 & 2.0 & 2.0 & 3.0 & 3.0 & 4.0 & 4.0 & 5.0 \\
\hline Subject 19 & 2.0 & 3.0 & 2.0 & 3.0 & 2.0 & 4.0 & 5.0 \\
\hline Subject 20 & 1.0 & 3.0 & 2.0 & 4.0 & 5.0 & 2.0 & 1.0 \\
\hline
\end{tabular}

\section{APPENDIX 3}

Table 3. Appendix C, shows the scores obtained in the subjective evaluations of 3 questions for dryer $\mathrm{A}, \mathrm{B}$ and $\mathrm{C}$.

\begin{tabular}{|l|c|c|c|c|c|c|c|c|c||}
\hline & QA1 & QA2 & QA3 & QB1 & QB2 & QB3 & QC1 & QC2 & QC3 \\
\hline Subject 1 & 1.0 & 2.0 & 3.0 & 3.0 & 4.0 & 3.0 & 1.0 & 0.0 & 3.0 \\
\hline Subject 2 & 10.0 & 9.0 & 9.0 & 2.0 & 3.0 & 1.0 & 2.0 & 3.0 & 1.0 \\
\hline Subject 3 & 1.0 & 2.0 & 3.0 & 3.0 & 2.0 & 1.0 & 3.0 & 2.0 & 1.0 \\
\hline Subject 4 & 3.0 & 2.0 & 1.0 & 10.0 & 9.0 & 10.0 & 4.0 & 1.0 & 2.0 \\
\hline Subject 5 & 3.0 & 4.0 & 5.0 & 1.0 & 1.0 & 3.0 & 10.0 & 9.0 & 9.0 \\
\hline Subject 6 & 1.0 & 4.0 & 5.0 & 1.0 & 3.0 & 2.0 & 2.0 & 1.0 & 2.0 \\
\hline Subject 7 & 2.0 & 3.0 & 1.0 & 2.0 & 0.0 & 1.0 & 1.0 & 0.0 & 1.0 \\
\hline Subject 8 & 1.0 & 2.0 & 3.0 & 1.0 & 2.0 & 3.0 & 1.0 & 2.0 & 3.0 \\
\hline Subject 9 & 3.0 & 3.0 & 1.0 & 3.0 & 0.0 & 1.0 & 2.0 & 0.0 & 1.0 \\
\hline Subject 10 & 2.0 & 2.0 & 4.0 & 2.0 & 1.0 & 4.0 & 3.0 & 1.0 & 4.0 \\
\hline Subject 11 & 3.0 & 4.0 & 0.0 & 3.0 & 1.0 & 0.0 & 4.0 & 1.0 & 0.0 \\
\hline Subject 12 & 1.0 & 1.0 & 3.0 & 1.0 & 0.0 & 3.0 & 0.0 & 0.0 & 3.0 \\
\hline Subject 13 & 2.0 & 0.0 & 3.0 & 2.0 & 0.0 & 3.0 & 2.0 & 0.0 & 3.0 \\
\hline Subject 14 & 1.0 & 4.0 & 0.0 & 1.0 & 3.0 & 0.0 & 1.0 & 2.0 & 0.0 \\
\hline Subject 15 & 2.0 & 3.0 & 1.0 & 2.0 & 0.0 & 1.0 & 1.0 & 0.0 & 1.0 \\
\hline Subject 16 & 1.0 & 2.0 & 1.0 & 1.0 & 2.0 & 1.0 & 0.0 & 2.0 & 1.0 \\
\hline Subject 17 & 2.0 & 3.0 & 1.0 & 2.0 & 4.0 & 1.0 & 10.0 & 9.0 & 10.0 \\
\hline Subject 18 & 2.0 & 2.0 & 3.0 & 9.0 & 8.0 & 10.0 & 0.0 & 2.0 & 1.0 \\
\hline Subject 19 & 2.0 & 3.0 & 0.0 & 2.0 & 3.0 & 0.0 & 1.0 & 3.0 & 0.0 \\
\hline Subject 20 & 2.0 & 2.0 & 3.0 & 2.0 & 0.0 & 3.0 & 3.0 & 0.0 & 3.0 \\
\hline
\end{tabular}

Table 4. Appendix C, shows the scores obtained in the subjective evaluations of 3 questions for dryer A, B and C.

\begin{tabular}{||c|c|c|c|c|c|}
\hline Questions & 1 & 2 & 3 & 4 & 5 \\
\hline Subject 1 & 1.0 & 2.0 & 3.0 & 2.0 & 0.0 \\
\hline Subject 2 & 10.0 & 9.0 & 9.0 & 10.0 & 9.0 \\
\hline Subject 3 & 1.0 & 2.0 & 3.0 & 4.0 & 1.0 \\
\hline Subject 4 & 3.0 & 2.0 & 1.0 & 1.0 & 0.0 \\
\hline Subject 5 & 3.0 & 4.0 & 0.0 & 2.0 & 3.0 \\
\hline Subject 6 & 1.0 & 4.0 & 1.0 & 5.0 & 1.0 \\
\hline Subject 7 & 2.0 & 0.0 & 1.0 & 4.0 & 4.0 \\
\hline Subject 8 & 1.0 & 2.0 & 3.0 & 3.0 & 1.0 \\
\hline Subject 9 & 3.0 & 3.0 & 1.0 & 2.0 & 5.0 \\
\hline Subject 10 & 2.0 & 2.0 & 4.0 & 1.0 & 2.0 \\
\hline Subject 11 & 3.0 & 4.0 & 0.0 & 2.0 & 2.0 \\
\hline Subject 12 & 1.0 & 1.0 & 3.0 & 2.0 & 3.0 \\
\hline Subject 13 & 2.0 & 1.0 & 1.0 & 0.0 & 0.0 \\
\hline Subject 14 & 1.0 & 4.0 & 0.0 & 1.0 & 4.0 \\
\hline Subject 15 & 2.0 & 3.0 & 1.0 & 4.0 & 2.0 \\
\hline Subject 16 & 1.0 & 2.0 & 1.0 & 5.0 & 1.0 \\
\hline Subject 17 & 2.0 & 3.0 & 1.0 & 5.0 & 3.0 \\
\hline Subject 18 & 2.0 & 2.0 & 3.0 & 3.0 & 5.0 \\
\hline Subject 19 & 2.0 & 3.0 & 0.0 & 2.0 & 0.0 \\
\hline Subject 20 & 2.0 & 2.0 & 3.0 & 5.0 & 0.0 \\
\hline
\end{tabular}

